\title{
A SIXTH-ORDER FINITE VOLUME METHOD FOR THE 1D BIHARMONIC OPERATOR: APPLICATION TO INTRAMEDULLARY NAIL SIMULATION
}

\author{
RiCARdo COSTA $^{a}$, GASPAR J. MACHADO $^{a, *}$, StÉPHANE CLAIN $^{a, b}$ \\ ${ }^{a}$ Centre of Mathematics \\ University of Minho, Campus de Azurém, 4800-058 Guimarães, Portugal \\ e-mail: pg24046@alunos.uminho.pt, \{gjm, clain\}@math.uminho.pt \\ ${ }^{b}$ Institute of Mathematics
}

Paul Sabatier University, 118, route de Narbonne, 31062 Toulouse, France

\begin{abstract}
A new very high-order finite volume method to solve problems with harmonic and biharmonic operators for onedimensional geometries is proposed. The main ingredient is polynomial reconstruction based on local interpolations of mean values providing accurate approximations of the solution up to the sixth-order accuracy. First developed with the harmonic operator, an extension for the biharmonic operator is obtained, which allows designing a very high-order finite volume scheme where the solution is obtained by solving a matrix-free problem. An application in elasticity coupling the two operators is presented. We consider a beam subject to a combination of tensile and bending loads, where the main goal is the stress critical point determination for an intramedullary nail.
\end{abstract}

Keywords: finite volume method, polynomial reconstruction operator, harmonic operator, biharmonic operator, high-order method.

\section{Introduction}

The finite volume method is a popular technique usually employed to carry out numerical approximations for conservation laws (see, for example, Kroner, 1997; Leveque, 2002; Audusse and Bristeau, 2007; Dumbser and Munz, 2007; Trangenstein, 2009).

In the 1990s, the finite volume method faced important developments to approximate elliptic and parabolic problems (see the work of Eymard et al. (2000) and the references therein), where second-order methods were designed for a large range of applications.

Recently, a new sixth-order finite volume method has been proposed for convection diffusion problems by Clain et al. (2013). The technique is based on specific polynomial reconstructions used for the fluxes (Hernández, 2002; Ollivier-Gooch and Altena, 2002; Toro and Hidalgo, 2009; Toro, 2009; Clain et al., 2011; Diot

*Corresponding author et al., 2011). We extend here the method to the biharmonic (or bi-Laplace) operator which is, up to our knowledge, the first attempt to design a finite volume scheme for a fourth-order differential operator. We restrict ourselves to the one-dimensional case to provide a simple but relevant context. We apply the proposed method to an example motivated by medical equipment modeling which couples the harmonic and biharmonic operators.

The organization of the paper is the following. Section 2 is devoted to the harmonic operator, where we introduce the mesh, the generic finite volume formulation, and the polynomial reconstruction operator to design the high-order finite volume scheme. In Section 3, we present the biharmonic operator case and the corresponding schemes we designed. Numerical tests are carried out in Section 4 to assess the scheme's ability to provide high-order accuracy both for the harmonic and biharmonic operators. A practical application of the method in a simulation of an intramedullary nail is presented in Section 5. 


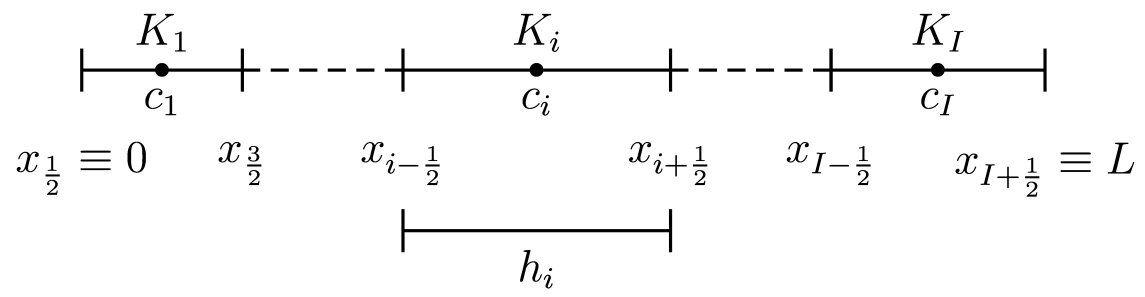

Fig. 1. Mesh and notation.

\section{Harmonic operator}

The harmonic operator is an essential building block for modeling phenomena such as elasticity, electromagnetism, and steady-state heat transfer, among others. In the one dimensional context, the harmonic (or Poisson) operator equation is

$$
\left(-\lambda \phi^{\prime}(x)\right)^{\prime}=f(x), \quad x \in \Omega,
$$

in a domain $\bar{\Omega}=\overline{(0, L)}$ with $L \in \mathbb{R}^{+}$, where $\lambda>0$ is assumed to be constant and $f$ stands for the source term. The harmonic equation is equipped with appropriate conditions on the boundary of the domain. In the present study we consider the following boundary conditions:

$$
\begin{aligned}
\phi(0) & =\phi_{\ell} \in \mathbb{R}, \\
\phi(L) & =\phi_{r} \in \mathbb{R}, \\
-\lambda \phi^{\prime}(L) & =F \in \mathbb{R},
\end{aligned}
$$

and we will examine two different situations: we prescribe (i) 2a and (2b), or (ii) 2a and (2c).

2.1. Mesh and discretization. To design the finite volume scheme, we introduce meshes $\mathcal{T}_{h}$ of $\Omega$, with $h$ as the mesh parameter, constituting of $I$ cells $K_{i}=$ $\left[x_{i-\frac{1}{2}}, x_{i+\frac{1}{2}}\right], i=1, \ldots, I$, of centroid $c_{i}$, where $x_{\frac{1}{2}}=$ $0, x_{I+\frac{1}{2}}=L, x_{i+\frac{1}{2}}=x_{i-\frac{1}{2}}+h_{i}$ are the interfaces (cf. Fig. 1) and $h$ is the maximum of the cell lengths. Integration of Eqn. (1) over cell $K_{i}$ yields

$$
\frac{1}{h_{i}}\left(F_{i+\frac{1}{2}}-F_{i-\frac{1}{2}}\right)=\bar{f}_{i}, \quad i=1, \ldots, I,
$$

where the fluxes are given by

$$
F_{i \pm \frac{1}{2}}=-\lambda \phi^{\prime}\left(x_{i \pm \frac{1}{2}}\right)
$$

and the mean source term by

$$
\bar{f}_{i}=\frac{1}{h_{i}} \int_{K_{i}} f(\xi) \mathrm{d} \xi
$$

Let $\phi_{i}$ be an approximation of the mean value of $\phi$ over $K_{i}$ and let us gather all the approximations in a vector $\Phi=\left(\phi_{i}\right)_{i=1, \ldots, I}$. To design a numerical scheme, we replace the exact fluxes $F_{i \pm \frac{1}{2}}$ with numerical fluxes up to a sixth-order accuracy $\mathcal{F}_{i \pm \frac{1}{2}}(\Phi)$ depending on the vector $\Phi$. In the same way, we also approximate the exact mean source term $\bar{f}_{i}$ by $f_{i}$ using a sixth-order Gaussian quadrature. The finite volume scheme cast in the residual form is

$$
\mathbb{F}_{i}(\Phi)=\frac{1}{h_{i}}\left(\mathcal{F}_{i+\frac{1}{2}}(\Phi)-\mathcal{F}_{i-\frac{1}{2}}(\Phi)\right)-f_{i}
$$

2.2. Polynomial reconstruction operator. We now turn to the critical point of the design of very high-order numerical fluxes. The technique proposed by Clain et al. (2013) for the two-dimensional elliptic problem is here adapted to the one-dimensional case where local polynomial approximations of the underlying solution are evaluated.

In the first stage, we define the stencils associated with the cells and the interfaces. For any cell $K_{i}, i=$ $1, \ldots, I$, we denote by $\widehat{S}_{i}$ the associated stencil composed of the $n$ closest neighbor cells (excluding cell $K_{i}$ ). In the same way, we denote by $\widehat{S}_{\frac{1}{2}}$ and $\widehat{S}_{I+\frac{1}{2}}$ the stencils constituted of the $n$ neighbor cells for outer interfaces $x_{\frac{1}{2}}=0$ and $x_{I+\frac{1}{2}}=L$, respectively. The second stage consists in defining the polynomial reconstructions based on the entries of the vector $\Phi$ associated with the appropriate stencils. We detail this in the following subsections.

2.2.1. Polynomial reconstruction on cells. Let $i \in$ $\{1, \ldots, I\}$ and $\phi_{i}$ be an approximation of the mean value of $\phi$ over cell $K_{i}$. We define the polynomial conservative reconstruction of degree $d$ as (see Clain et al., 2013)

$$
\phi_{i}(x ; d)=\phi_{i}+\sum_{\alpha=1}^{d} \mathcal{R}_{i, \alpha}\left[\left(x-c_{i}\right)^{\alpha}-M_{i, \alpha}\right] \text {, }
$$

where we have set $M_{i, \alpha}=\frac{1}{h_{i}} \int_{K_{i}}\left(x-x_{i}\right)^{\alpha} \mathrm{d} x$ and the vector $\mathcal{R}_{i}$ gathers the polynomial coefficients $\mathcal{R}_{i, \alpha}$, $\alpha=1, \ldots, d$. For a given stencil $\widehat{S}_{i}$ and positive weights 
$\left(\omega_{i, j}\right)_{j=1, \ldots, \# \widehat{S}_{i}}$, we consider the quadratic functional

$$
\widehat{E}_{i}\left(\mathcal{R}_{i}\right)=\sum_{j \in \widehat{S}_{i}} \omega_{i, j}\left[\frac{1}{h_{j}} \int_{K_{j}} \phi_{i}(x ; \mathrm{d}) \mathrm{d} x-\phi_{j}\right]^{2} .
$$

We denote by $\widehat{\mathcal{R}}_{i}$ the unique vector that minimizes the quadratic functional and set $\widehat{\phi}_{i}(x ; d)$ as the associated polynomial function that corresponds to the best approximation in the least squares sense of the data of the stencil.

\subsubsection{Polynomial reconstruction at the outer inter-}

faces. For the left boundary interface $x_{\frac{1}{2}}$, we adapt the previous polynomial reconstruction in order to obtain the polynomial $\phi_{\frac{1}{2}}(x ; d)$ as

$$
\phi_{\frac{1}{2}}(x ; d)=\phi_{\ell}+\sum_{\alpha=1}^{d} \widehat{\mathcal{R}}_{\frac{1}{2}, \alpha}\left(x-x_{\frac{1}{2}}\right)^{\alpha},
$$

where vector $\mathcal{R}_{\frac{1}{2}}$ gathers the polynomial coefficients $\mathcal{R}_{\frac{1}{2}, \alpha}, \alpha=1, \ldots, d$. For a given stencil $\widehat{S}_{\frac{1}{2}}$ and positive

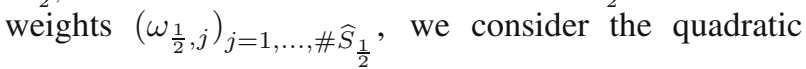
functional

$$
\widehat{E}_{\frac{1}{2}}\left(\mathcal{R}_{\frac{1}{2}}\right)=\sum_{j \in \widehat{S}_{\frac{1}{2}}} \omega_{\frac{1}{2}, j}\left[\frac{1}{h_{j}} \int_{K_{j}} \phi_{\frac{1}{2}}(x ; d) \mathrm{d} x-\phi_{j}\right]^{2} .
$$

We denote by $\widehat{\mathcal{R}}_{\frac{1}{2}}$ the unique vector which minimizes the quadratic functional and set $\widehat{\phi}_{\frac{1}{2}}(x ; d)$ as the associated polynomial function that corresponds to the best approximation in the least squares sense of the data of the stencil.

We proceed in the same way for the polynomial $\widehat{\phi}_{I+\frac{1}{2}}(x ; d)$ associated with the interface $x_{I+\frac{1}{2}}$.

2.3. High-order fluxes. Having all the polynomial reconstructions in hand, we detail the numerical fluxes for the harmonic operator with respect to the interfaces:

- for an inner interface the flux is given by

$$
\begin{array}{r}
\mathcal{F}_{i+\frac{1}{2}}=-\lambda \frac{\widehat{\phi}_{i}^{\prime}\left(x_{i+\frac{1}{2}}\right)+\widehat{\phi}_{i+1}^{\prime}\left(x_{i+\frac{1}{2}}\right)}{2 \quad}, \\
i=1, \ldots, I-1 ;
\end{array}
$$

- the flux on the left boundary interface is given by

$$
\mathcal{F}_{\frac{1}{2}}=-\lambda \widehat{\phi}_{\frac{1}{2}}^{\prime}(0)
$$

- the flux on the right boundary interface is given by

$$
\mathcal{F}_{I+\frac{1}{2}}=-\lambda \widehat{\phi}_{I+\frac{1}{2}}^{\prime}(L)
$$

for the condition (2b) or by

$$
\mathcal{F}_{I+\frac{1}{2}}=F
$$

for the case (2c)

Since $\mathcal{F}_{i+\frac{1}{2}}$ linearly depends on the vector $\Phi$, the residual operator $\Phi \rightarrow \mathbb{F}_{i}(\Phi)$ is an affine operator. Gathering all the components of the residual in vector $\mathbb{F}(\Phi)$, we obtain an affine operator from $\mathbb{R}^{I}$ in $\mathbb{R}^{I}$ such that the vector $\Phi^{\star}$, a solution of the problem $\mathbb{F}(\Phi)=$ $0_{I}$, provides a constant piecewise approximation. The solution is very accurate since the reconstruction process based on the value of the vector $\Phi^{\star}$ gives polynomial approximations up to the sixth order. Notice that the method is matrix-free and the linear problem is solved by applying a GMRES procedure as explained by Clain et al. (2013).

\section{Biharmonic operator}

The biharmonic operator (also called the bi-Laplacian operator) equation is

$$
\left(-\mu \psi^{\prime \prime}(x)\right)^{\prime \prime}=g(x), \quad x \in \Omega,
$$

in a domain $\bar{\Omega}=\overline{(0, L)}$ with $L \in \mathbb{R}^{+}$, where $\mu>$ 0 is assumed to be constant and $g$ is the source term. The biharmonic equation is equipped with appropriate conditions on the boundary of the domain. In the present study we consider the following boundary conditions:

$$
\begin{aligned}
\psi(0) & =\psi_{\ell} \in \mathbb{R}, \\
\psi(L) & =\psi_{r} \in \mathbb{R}, \\
\psi^{\prime}(0) & =\psi_{\ell \ell} \in \mathbb{R}, \\
\psi^{\prime}(L) & =\psi_{r r} \in \mathbb{R}, \\
-\mu \psi^{\prime \prime}(0) & =M_{\ell} \in \mathbb{R}, \\
-\mu \psi^{\prime \prime}(L) & =M_{r} \in \mathbb{R}, \\
-\mu \psi^{\prime \prime \prime}(L) & =G \in \mathbb{R},
\end{aligned}
$$

and will examine three different situations: we prescribe (i) 4a , 4b), 4c), and 4d), or (ii) 4a , 4b), 4e), and (4f), or (iii) 4a , 4c), 4f), and 4g).

3.1. Discretization. The mesh and the finite volume discretization follow as in Section 2.1 and integration of Eqn. (3) over cell $K_{i}$ yields

$$
\frac{1}{h_{i}}\left(G_{i+\frac{1}{2}}-G_{i-\frac{1}{2}}\right)=\bar{g}_{i}, \quad i=1, \ldots, I,
$$

where the fluxes are given by $G_{i \pm \frac{1}{2}}=-\mu \psi^{\prime \prime \prime}\left(x_{i \pm \frac{1}{2}}\right)$ and the mean source term by $\bar{g}_{i}=\frac{1}{h_{i}} \int_{K_{i}} g(\xi) \mathrm{d} \xi$. We 
replace the exact fluxes on interfaces $G_{i \pm \frac{1}{2}}$ by a numerical flux $\mathcal{G}_{i \pm \frac{1}{2}}(\Psi)$ up to the sixth-order accuracy based on the vector $\Psi=\left(\psi_{i}\right)_{i=1, \ldots, I}$ gathering approximations of the mean values of $\psi$. As in the previous section, the exact mean source term $\bar{g}_{i}$ is approximated by a sixth-order Gaussian quadrature denoted by $g_{i}$ and the finite volume scheme cast in the residual form is

$$
\mathbb{G}_{i}(\Psi)=\frac{1}{h_{i}}\left(\mathcal{G}_{i+\frac{1}{2}}(\Psi)-\mathcal{G}_{i-\frac{1}{2}}(\Psi)\right)-g_{i} .
$$

3.2. Polynomial reconstruction operator. A delicate point is the introduction of the boundary conditions in the reconstructions. Indeed, one has to handle two conditions at each outer interfaces, but it is not possible to support the two conditions on the same function, namely, on functions $\widehat{\boldsymbol{\psi}}_{\frac{1}{2}}(x ; d)$ and $\widehat{\boldsymbol{\psi}}_{I+\frac{1}{2}}(x ; d)$. As a consequence, functions $\widehat{\psi}_{1}(x ; d)$ or $\widehat{\psi}_{I}(x ; d)$ will also support some boundary conditions to provide an invertible linear system.

\subsubsection{Polynomial reconstruction on inner cells.} For cells $K_{i}, i=2, \ldots, I-1$, the reconstructions are performed exactly as in Section 2.2.1 and provide polynomial functions $\widehat{\psi}_{i}(x ; d)$.

3.2.2. Polynomial reconstruction at the outer interfaces. To take into account the boundary conditions (4a) and (4b), we reconstruct the polynomials $\widehat{\psi}_{\frac{1}{2}}(x ; d)$ and $\widehat{\psi}_{I+\frac{1}{2}}(x ; d)$, respectively, as described in Section 2.2.2

\subsubsection{Polynomial reconstruction on the first and} the last cell. We reach the important point where we shall construct the polynomial functions $\widehat{\psi}_{1}(x ; d)$ and $\widehat{\psi}_{I}(x ; d)$ in order to take into account the boundary conditions (4c)-4ed and 4d - (4f), respectively.

Let $\psi_{1}$ be an approximation of the mean value of $\psi$ over cell $K_{1}$. The conservative polynomial reconstruction associated with the first cell $K_{1}$ is

$$
\boldsymbol{\psi}_{1}(x ; d)=\psi_{1}+\sum_{\alpha=1}^{\mathrm{d}} \widehat{\mathcal{R}}_{i, \alpha}\left[\left(x-c_{1}\right)^{\alpha}-M_{1, \alpha}\right],
$$

where the vector $\mathcal{R}_{1}$ gathers the polynomial coefficients $\mathcal{R}_{1, \alpha}, \alpha=1, \ldots, d$. For a given stencil $\widehat{S}_{1}$ and positive

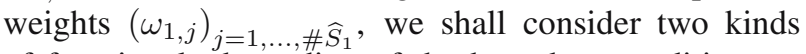
of functionals depending of the boundary condition we shall apply. To introduce the condition $4 \mathrm{c}$, the quadratic functional becomes

$$
\begin{aligned}
\widehat{E}_{1}\left(\mathcal{R}_{1}\right)= & \sum_{j \in \widehat{S}_{1}} \omega_{1, j}\left[\frac{1}{h_{j}} \int_{K_{j}} \psi_{1}(x ; d) \mathrm{d} x-\psi_{j}\right]^{2} \\
& +\left[\psi_{1}^{\prime}(0)-\psi_{\ell \ell}\right]^{2}
\end{aligned}
$$

and in order to consider the condition (4e) we set

$$
\begin{aligned}
\widehat{E}_{1}\left(\mathcal{R}_{1}\right)= & \sum_{j \in \widehat{S}_{1}} \omega_{1, j}\left[\frac{1}{h_{j}} \int_{K_{j}} \boldsymbol{\psi}_{1}(x ; d) \mathrm{d} x-\psi_{j}\right]^{2} \\
& +\left[\psi_{1}^{\prime \prime}(0)+\frac{M_{\ell}}{\mu}\right]^{2} .
\end{aligned}
$$

We denote by $\widehat{\mathcal{R}}_{1}$ the unique vector which minimizes the quadratic functional (5) for the boundary condition (4c) and the quadratic functional (6) for the boundary condition (4e), and set $\widehat{\boldsymbol{\psi}}_{1}(x ; d)$ as the associated polynomial function.

We proceed in the same way for the polynomial $\widehat{\psi}_{I}(x ; d)$ associated to the last cell $K_{I}$.

3.3. High-order finite volumes scheme. Having in hand all the polynomial reconstructions, we compute the fluxes for the biharmonic operator:

- for an inner interface, the flux is

$$
\begin{aligned}
& \mathcal{G}_{i+\frac{1}{2}}=-\mu \frac{\widehat{\boldsymbol{\psi}}_{i}^{\prime \prime \prime}\left(x_{i+\frac{1}{2}}\right)+\widehat{\boldsymbol{\psi}}_{i+1}^{\prime \prime \prime}\left(x_{i+\frac{1}{2}}\right)}{2}, \\
& i=1, \ldots, I-1,
\end{aligned}
$$

- on the left boundary, one has

$$
\mathcal{G}_{\frac{1}{2}}=-\mu \widehat{\boldsymbol{\psi}}_{\frac{1}{2}}^{\prime \prime \prime}(0),
$$

- for the right boundary, we prescribe

$$
\mathcal{G}_{I+\frac{1}{2}}=-\mu \widehat{\boldsymbol{\psi}}_{I+\frac{1}{2}}^{\prime \prime \prime}(L)
$$

for the condition $4 \mathrm{~b}$ or

$$
\mathcal{G}_{I+\frac{1}{2}}=G
$$

for the condition $4 \mathrm{~g}$.

\section{Numerical results}

Quantitative and qualitative assessments of the scheme's robustness and accuracy both for the harmonic and the biharmonic operator are addressed in this section. To evaluate the error between the exact solution and the numerical solution, we introduce the $L^{\infty}$ norms

$$
\begin{aligned}
& E_{\infty}(I)=\max _{i=1}^{I}\left|\phi_{i}-\bar{\phi}_{i}\right|, \\
& E_{\infty}(I)=\max _{i=1}^{I}\left|\psi_{i}-\bar{\psi}_{i}\right|,
\end{aligned}
$$

where $\bar{\phi}_{i}$ and $\bar{\psi}_{i}$ are the exact mean values of $\phi$ and $\psi$, respectively, over cell $K_{i}$. The orders of convergence based on the $L^{\infty}$-norms, given by

$$
O_{\infty}\left(I_{1}, I_{2}\right)=\frac{\mid \log \left(E_{\infty}\left(I_{1}\right) /\left(E_{\infty}\left(I_{2}\right)\right) \mid\right.}{\left|\log \left(I_{1} / I_{2}\right)\right|},
$$


are also provided. The notation $\mathbb{P}_{\mathrm{d}}(n)$ means that we employ $d$-degree polynomial reconstructions involving $n$ cells stencils. The weights we will consider are summarized with the notation $\omega_{i, j}=q \mid r, q, r \in \mathbb{R}^{+}$, with the following meaning: if $i$ and $j$ are contiguous cells, then $\omega_{i, j}=q$; otherwise, $\omega_{i, j}=r$ (this notation extends in a natural way for the cases $\omega_{\frac{1}{2}, j}$ and $\omega_{I+\frac{1}{2}, j}$ ). In the present study, all the computations have been carried out with weights $3 \mid 1$ for stability reasons. Moreover, to reduce the computational effort, a preconditioning matrix is used as proposed by Clain et al. (2013) for steady-state problems as well as when dealing with time-dependent implicit schemes.

Example 1. We first assess the numerical scheme's accuracy for the harmonic operator equipped with the boundary conditions (2a) and (2b). Taking $f(x)=e^{x}$ and $\lambda=1$, the exact solution is $\phi(x)=-e^{x}+(e-1) x+1$. In Table 1 we report the errors and convergence rates between the numerical solution and the exact one. The effective second-, fourth-, and sixth orders of convergence are achieved when dealing with the $\mathbb{P}_{1}(2), \mathbb{P}_{3}(4)$, and $\mathbb{P}_{5}(6)$ reconstructions, respectively. Notice that the sixth-order scheme exceeds the IEEE-754 standard capacity of double for the 160 cells mesh due to the very high accuracy of the reconstruction technique.

Example 2. Another example for the harmonic operator concerns the Dirichlet-Neumann boundary conditions (2a) and (2c). Taking $f(x)=e^{x}$ and $\lambda=1$, we get $F=1$ and the exact solution becomes $\phi(x)=$ $-e^{x}+(e-1) x+1$.

As in the previous case, Table 2 shows that the scheme achieved effective second-, fourth-, and sixth-orders of convergence for the $\mathbb{P}_{1}(2), \mathbb{P}_{3}(4)$, and $\mathbb{P}_{5}(6)$ polynomial reconstructions, respectively. Notice that Tables 1 and 2 provide similar errors and that the IEEE-754 standard limitation is reached for too finer meshes.

Example 3. We now turn to the biharmonic operator. Taking $\mu=1$ and $g(x)=e^{x}$, the exact solution becomes $\psi(x)=-e^{x}-(e-3) x^{3}-(5-2 e) x^{2}+x+1$. Thanks to the exact solution, we prescribe the boundary conditions 4a), (4b), 4c), and 4d).

Table 3 reports the convergence orders and the scheme's accuracy for the $\mathbb{P}_{3}(4), \mathbb{P}_{5}(6)$, and $\mathbb{P}_{7}(8)$ polynomial reconstructions. We observe some differences with respect to the harmonic operator. For example, the scheme hardly achieved an effective sixth-order convergence rate with the fifth-degree polynomial reconstruction (slightly larger than 5) in contrast to the harmonic case, which delivers the optimal order. Using a seventh-degree polynomial reconstruction, the IEEE-754 standard limitation is, one more time, patent.
Example 4. We deal with a similar situation taking $\mu=1$ and $g(x)=e^{x}$, but Neumann conditions are now prescribed. The exact solution is $\psi(x)=-e^{x}+$ $\left(\frac{e-1}{6}\right) x^{3}+x^{2}+\left(\frac{5 e-11}{6}\right) x+1$, and we consider the approximation of the biharmonic operator equipped with the boundary conditions 4a , 4b), 4e), and (4f).

In Table 4 we display the errors and convergence orders. Clear differences appears with respect to the former case. The scheme only reached sixth-order accuracy with the $\mathbb{P}_{7}(8)$ reconstruction. Unlike the harmonic operator, the convergence orders for the biharmonic operator differ with the choice of the boundary conditions. Comparisons between Tables 3 and 4 emphasize such differences. As an example, the $\mathbb{P}_{3}(4)$ reconstruction achieves an effective third-order accuracy for Example 3. whereas the same reconstruction only provides a second-order accuracy for Example 4.

Example 5. The last test we address concerns the biharmonic operator equipped with the boundary conditions (4a), (4c), (4f), and (4g). The exact solution is $\psi(x)=-e^{x}+\left(\frac{e-1}{6}\right) x^{3}+x^{2}+x+1$ with $g(x)=e^{x}$, $\mu=1$, and $G=1$.

Errors and convergence rates are shown in Table 5 where we observe effective second-, fourth-, and sixth orders of convergence for the $\mathbb{P}_{3}(4), \mathbb{P}_{5}(6)$, and $\mathbb{P}_{7}(8)$ reconstructions, respectively. The high preconditioning number (no preconditioning matrix has been employed in the numerical simulations) associated with the IEEE-754 limitation is responsible for the low threshold of the error saturation (around $10^{-10}$ ).

\section{Study case}

Numerical simulation of the intramedullary nail stress (a metal rod used to treat fractures of long bones of the body such as the femur or the tibia) is an interesting example, where one has to couple the harmonic and biharmonic operators. The device attempts to stabilize and align the fracture until full consolidation of the bone submitted to internal stresses deriving from the traction or pressure applied on the bone.

5.1. Model. In Fig.2 (Ramos and Simoes, 2009), four different types of femoral fractures and the application of an intramedullary nail are shown. As a test case, we shall consider the first two configurations displayed in Fig. 2.

We aim to numerically determine the stress critical point (or the maximum stress point) of an intramedullary nail since rupture occurs when the stress exceeds the ultimate tensile strength of the material. Such a prediction is of great importance to design robust and resistant medical devices to avoid rupture under normal situations. For the sake of simplicity, we represent the body of the intramedullary nail by a rectangle, as displayed in 
Table 1. Results for Example 1

\begin{tabular}{|c||c|c||c|c||c|c|}
\hline \multirow{2}{*}{$I$} & \multicolumn{2}{c||}{$\mathbb{P}_{1}(2)$} & \multicolumn{2}{c||}{$\mathbb{P}_{3}(4)$} & \multicolumn{2}{c|}{$\mathbb{P}_{5}(6)$} \\
\cline { 2 - 7 } & $E_{\infty}$ & $O_{\infty}$ & $E_{\infty}$ & $O_{\infty}$ & $E_{\infty}$ & $O_{\infty}$ \\
\hline \hline 20 & $6.92 \mathrm{E}-03$ & - & $2.14 \mathrm{E}-06$ & - & $5.18 \mathrm{E}-09$ & - \\
40 & $1.82 \mathrm{E}-03$ & 1.93 & $1.44 \mathrm{E}-07$ & 3.89 & $8.88 \mathrm{E}-11$ & 5.86 \\
80 & $4.66 \mathrm{E}-04$ & 1.96 & $9.39 \mathrm{E}-09$ & 3.94 & $1.46 \mathrm{E}-12$ & 5.93 \\
160 & $1.18 \mathrm{E}-04$ & 1.98 & $5.99 \mathrm{E}-10$ & 3.97 & $7.83 \mathrm{E}-14$ & 4.22 \\
\hline
\end{tabular}

Table 2. Results for Example 2

\begin{tabular}{|c||c|c||c|c||c|c|}
\hline \multirow{2}{*}{$I$} & \multicolumn{2}{c||}{$\mathbb{P}_{1}(2)$} & \multicolumn{2}{c||}{$\mathbb{P}_{3}(4)$} & \multicolumn{2}{c|}{$\mathbb{P}_{5}(6)$} \\
\cline { 2 - 7 } & $E_{\infty}$ & $O_{\infty}$ & $E_{\infty}$ & $O_{\infty}$ & $E_{\infty}$ & $O_{\infty}$ \\
\hline \hline 20 & $5.70 \mathrm{E}-03$ & - & $1.66 \mathrm{E}-06$ & - & $2.85 \mathrm{E}-09$ & - \\
40 & $1.50 \mathrm{E}-03$ & 1.93 & $1.07 \mathrm{E}-07$ & 3.96 & $4.18 \mathrm{E}-11$ & 6.09 \\
80 & $3.83 \mathrm{E}-04$ & 1.97 & $6.77 \mathrm{E}-09$ & 3.98 & $6.80 \mathrm{E}-13$ & 5.94 \\
160 & $9.69 \mathrm{E}-05$ & 1.98 & $4.26 \mathrm{E}-10$ & 3.99 & $1.89 \mathrm{E}-13$ & 1.85 \\
\hline
\end{tabular}

Table 3. Results for Example 3

\begin{tabular}{|c||c|c||c|c||c|c|}
\hline \multicolumn{1}{|c||}{$I$} & \multicolumn{2}{c||}{$\mathbb{P}_{3}(4)$} & \multicolumn{2}{c||}{$\mathbb{P}_{5}(6)$} & \multicolumn{2}{c|}{$\mathbb{P}_{7}(8)$} \\
\cline { 2 - 7 } & $E_{\infty}$ & $O_{\infty}$ & $E_{\infty}$ & $O_{\infty}$ & $E_{\infty}$ & $O_{\infty}$ \\
\hline \hline 20 & $9.75 \mathrm{e}-05$ & - & $7.32 \mathrm{e}-08$ & - & $5.23 \mathrm{e}-10$ & - \\
40 & $1.21 \mathrm{e}-05$ & 3.01 & $2.11 \mathrm{e}-09$ & 5.11 & $4.50 \mathrm{e}-12$ & 6.86 \\
80 & $1.42 \mathrm{e}-06$ & 3.10 & $6.79 \mathrm{e}-11$ & 4.96 & $5.65 \mathrm{e}-14$ & 6.31 \\
160 & $1.49 \mathrm{e}-07$ & 3.25 & $1.78 \mathrm{e}-12$ & 5.25 & $6.08 \mathrm{e}-13$ & - \\
\hline
\end{tabular}

Table 4. Results for Example 4

\begin{tabular}{|c||c|c||c|c||c|c|}
\hline \multicolumn{1}{|c||}{$I$} & \multicolumn{2}{c||}{$\mathbb{P}_{3}(4)$} & \multicolumn{2}{c||}{$\mathbb{P}_{5}(6)$} & \multicolumn{2}{c|}{$\mathbb{P}_{7}(8)$} \\
\cline { 2 - 7 } & $E_{\infty}$ & $O_{\infty}$ & $E_{\infty}$ & $O_{\infty}$ & $E_{\infty}$ & $O_{\infty}$ \\
\hline \hline 20 & $8.02 \mathrm{E}-04$ & - & $3.04 \mathrm{E}-06$ & - & $1.88 \mathrm{E}-08$ & - \\
40 & $1.94 \mathrm{E}-04$ & 2.05 & $1.94 \mathrm{E}-07$ & 3.97 & $3.04 \mathrm{E}-10$ & 5.95 \\
80 & $4.75 \mathrm{E}-05$ & 2.03 & $1.23 \mathrm{E}-08$ & 3.98 & $1.92 \mathrm{E}-11$ & 3.98 \\
160 & $1.17 \mathrm{E}-05$ & 2.02 & $7.71 \mathrm{E}-10$ & 4.00 & $4.17 \mathrm{E}-11$ & - \\
\hline
\end{tabular}

Table 5. Results for Example 5

\begin{tabular}{|c||c|c||c|c||c|c|}
\hline \multirow{2}{*}{\multicolumn{1}{|c||}{$I$}} & \multicolumn{2}{c||}{$\mathbb{P}_{3}(4)$} & \multicolumn{2}{c||}{$\mathbb{P}_{5}(6)$} & \multicolumn{2}{c|}{$\mathbb{P}_{7}(8)$} \\
\cline { 2 - 7 } & $E_{\infty}$ & $O_{\infty}$ & $E_{\infty}$ & $O_{\infty}$ & $E_{\infty}$ & $O_{\infty}$ \\
\hline \hline 20 & $3.49 \mathrm{e}-03$ & - & $1.54 \mathrm{e}-05$ & - & $8.98 \mathrm{e}-08$ & - \\
40 & $9.11 \mathrm{e}-04$ & 1.94 & $1.07 \mathrm{e}-06$ & 3.84 & $1.60 \mathrm{e}-09$ & 5.81 \\
80 & $2.32 \mathrm{e}-04$ & 1.97 & $7.05 \mathrm{e}-08$ & 3.93 & $3.60 \mathrm{e}-10$ & 2.16 \\
160 & $5.85 \mathrm{e}-05$ & 1.99 & $8.15 \mathrm{e}-09$ & 3.11 & $1.29 \mathrm{e}-10$ & 1.48 \\
\hline
\end{tabular}




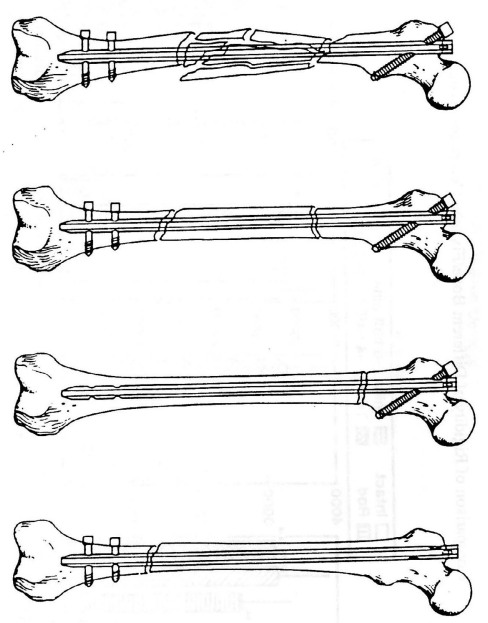

Fig. 2. Femoral fractures and the use of intramedullary nails.

Fig. 3 Usually, intramedullary nails have a ring-shaped cross-section and are made of stainless steel or titanium. The body has a small curvature, which we shall assume null in the present study, leading to a straight beam as the domain. We also state that there is a single resultant force $F_{0}$ in the upper screw and there is no rotational loads (cf. Fig. (3). We assume that the left edge of the intramedullary nail is fixed with two screws while two forces, $F_{1}$ and $F_{2}$, resulting from the decomposition of the force $F_{0}$ following the two axes are applied on the opposite edge. A bending moment $M$ is also prescribed on the right edge (cf. Fig. 3). In Fig. 4, the simplified unidimensional geometry we shall deal with is sketched out, where the employed forces and moment are represented.

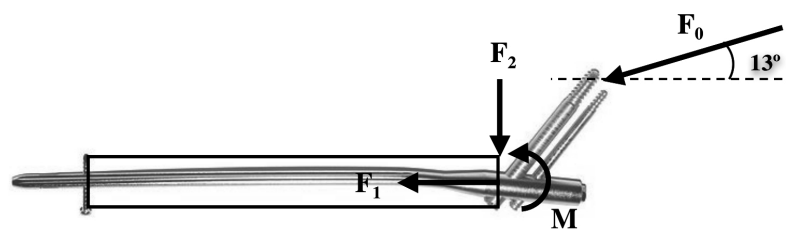

Fig. 3. Body of the intramedullary nail (bounded by a rectangle) and forces.

We briefly present the model where the two operators are coupled. Since there is no load in the $z$-direction and, therefore, the $z$-component of the stress vector vanishes, we only compute the stresses in the $x O y$ plane and the two-dimensional Cauchy stress tensor $[T]$ is given by

$$
[T]=\left[\begin{array}{cc}
\sigma_{x} & \tau_{x y} \\
\tau_{y x} & \sigma_{y}
\end{array}\right],
$$

where $\sigma$ for the normal stress while $\tau$ for the shear stress. We neglect $\tau_{x y}$ and $\tau_{y x}$ due to their small influence

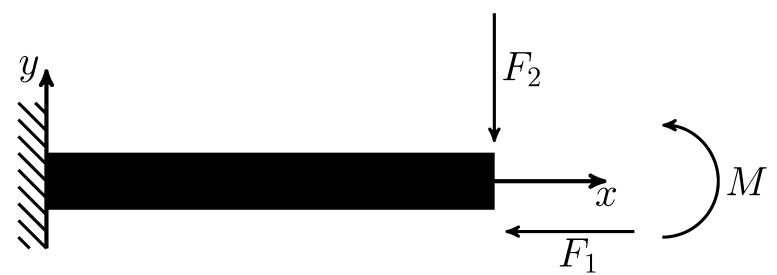

Fig. 4. Schematic representation of the intramedullary nail and forces.

(Branco, 2011), whereas $\sigma_{x}$ and $\sigma_{y}$ derive from Hooke's law for an isotropic material and are given by

$$
\sigma_{x}=\frac{E}{(1+v)(1-2 v)}\left[(1-v) \varepsilon_{x}+v\left(\varepsilon_{y}+\varepsilon_{z}\right)\right]
$$

and

$$
\sigma_{y}=\frac{E}{(1+v)(1-2 v)}\left[(1-v) \varepsilon_{y}+v\left(\varepsilon_{x}+\varepsilon_{z}\right)\right],
$$

with $E$ being Young's modulus and $v$ Poisson's ratio, while $\varepsilon_{x}, \varepsilon_{y}$, and $\varepsilon_{z}$ stand for the strain components in the $x$-, $y$-, and $z$-direction, respectively. Provided that $\varepsilon_{y}=\varepsilon_{z}=-v \varepsilon_{x}$, we deduce that $\sigma_{y}=0$.

Let $u=u(x)$ and $v=v(x)$ denote the horizontal and vertical displacements of the neutral axis of the beam. We assume that each cross-section of the beam remains orthogonal to the neutral axis (Euler-Bernoulli beam theory for small strains) such that the angular displacement of the cross-section is given by $\mathrm{d} v / \mathrm{d} x$. Therefore, the strain $\varepsilon_{x}$ in the $x O y$ plane writes

$$
\varepsilon_{x}(x, y)=\frac{\partial u(x)}{\partial x}-\frac{\partial^{2} v(x)}{\partial x^{2}} y .
$$

Let us consider a ring-shaped section beam of length $L$, where $D_{i}$ and $D_{o}$ are the inner and the outer diameter, respectively, i.e., $x \in[0, L]$ and $y \in\left[-D_{o} / 2,-D_{i} / 2\right] \cup$ $\left[D_{i} / 2, D_{o} / 2\right]$. One has to determine $u$ and $v$ to compute the strain component $\varepsilon_{x}$, and then to compute the stress component $\sigma_{x}$ with Eqn. (7).

5.2. Elastic beam theory. We now introduce mathematical models to compute the unknowns $u$ and $v$. The bending phenomenon occurs when an external load is applied perpendicularly to the longitudinal axis, whereas the tensile occurs when an external load is applied in the same direction as the longitudinal axis. It results that the bending to a vertical displacement of the beam while the tensile to a horizontal one. Since the total displacement is the sum of the displacements caused by each body force apart (mechanical superposition principle), we split the intramedullary nail problem into two subproblems: the tensile problem and the bending one. The first one 
deals with the load $F_{1}$ associated with the horizontal displacement $u$ and the second one with the loads $F_{2}$ and $M_{r}$ associated with the vertical displacement $v$. According elasticity theory, the tensile problem becomes

$$
\left(-E A u^{\prime}(x)\right)^{\prime}=0,
$$

where $A$ is the cross-sectional area of the beam. The equation is equipped with the boundary conditions

$$
\begin{aligned}
u(0) & =0, \\
-E A u^{\prime}(L) & =F_{1} .
\end{aligned}
$$

This problems is a specific case of the harmonic operator, where $\lambda=E A, \phi=u, f=0, F=F_{1}$, and $\phi_{\ell}=0$.

On the other hand, according to the Euler-Bernoulli theory, the bending problem is

$$
\left(-E I v^{\prime \prime}(x)\right)^{\prime \prime}=g,
$$

where $I$ stands for the second moment of area relatively to its longitudinal axis and perpendicular to the bending plane (in the case of a ring-shaped cross-section, the second moment of area is given by $\left.I=\frac{\pi}{16}\left(D_{o}^{2}-D_{i}^{2}\right)\right)$. The function $g$ is the vertical gravity applied along the $x$-axis, which we shall neglect in this practical application. The equation is equipped with the boundary conditions

$$
\begin{aligned}
v(0) & =0, \\
v^{\prime}(0) & =0, \\
-E I v^{\prime \prime}(L) & =M, \\
-E I v^{\prime \prime \prime}(L) & =F_{2} .
\end{aligned}
$$

This problems is a specific case of the biharmonic operator, where $\mu=E I, \psi=v, g=0, G=F_{2}, \psi_{\ell}=0$, and $\psi_{\ell \ell}=0$.

To perform the numerical simulation, we consider a stainless steel intramedullary nail with $20 \mathrm{~cm}$ of length, $5 \mathrm{~mm}$ of inner diameter, $11 \mathrm{~mm}$ of outer diameter, and the upper screw length of $5 \mathrm{~cm}$. Young's modulus and Poisson's ratio for stainless steel are $200 \mathrm{GPa}$ and 0.3 , respectively. According to Barreira et al. (2008), for a daily physical activity such as walking down stairs and for a person weighing $70 \mathrm{~kg}$, the maximum force applied in the femur head is about $1784 \mathrm{~N}$ with a direction shifted $13^{\circ}$ from the cortical plane. Assuming that $50 \%$ of this force is supported by the intramedullary nail, we evaluate $F_{1}=869.15 \mathrm{~N}, F_{2}=200.65 \mathrm{~N}$ and $M=$ $26.85 \mathrm{~N} \mathrm{~m}$. We considered a mesh of 80 cells and a fifth-degree polynomial reconstruction both for the tensile and the bending. Figure 5 shows the stress field of the longitudinal section of the intramedullary nail body. The stress critical point is located in the upper-right corner of the longitudinal section with a stress of $-436.8 \mathrm{MPa}$. The negative value means a compressive stress.

\section{Conclusions}

We presented an adaptation of the very high-order finite volume scheme introduced by Clain et al. (2013) for one-dimensional harmonic and biharmonic operators, where a specific discretization for each of these operators should be considered in order to obtain a sixth order of convergence. Numerical simulations have been carried out to assess the method's efficiency to provide a scheme with sixth order convergence. An application in the elasticity context has also been proposed to show that the presented method may be a future alternative to the classical finite element method.

\section{Acknowledgment}

This research was financed by FEDER funds through Programa Operacional Factores de Competitividade-COMPETE and by Portuguese Funds through FCT—Fundação para a Ciência e a Tecnologia, within the projects PEst-C/MAT/UI0013/2014 and PTDC/MAT/121185/2010 via a grant for research initiation (BII) awarded by the Centre of Mathematics of the School of Science at the University of Minho, Portugal. G.J. Machado and S. Clain were financed by Programa Pessoa Cooperação Transnacional Portugal/França Fundação para a Ciência e a Tecnologia with the reference 130631467126259 .

\section{References}

Audusse, E. and Bristeau, M.-O. (2007). Finite-volume solvers for a multilayer Saint-Venant system, International Journal of Applied Mathematics and Computer Science 17(3): 311-320, DOI: 10.2478/v10006-007-0025-0.

Barreira, L., Teixeira, C. and Fonseca, E. (2008). Avaliação da resistência do colo do fémur utilizando o modelo de elementos finitos, Revista da Associação Portuguesa de Análise Experimental de Tensões 16: 19-24.

Branco, C.M. (2011). Mecânica dos Materiais, Fundação Calouste Gulbenkian, Lisboa.

Clain, S., Diot, S. and Loubère, R. (2011). A high-order polynomial finite volume method for hyperbolic system of conservation laws with multi-dimensional optimal order detection (MOOD), Journal of Computational Physics 230(10): 4028-4050.

Clain, S., Machado, G.J., Nóbrega, J.M. and Pereira, R.M.S. (2013). A sixth-order finite volume method for the convection-diffusion problem with discontinuous coefficients, Computer Methods in Applied Mechanics and Engineering 267(1): 43-64.

Diot, S., Clain, S. and Loubère, R. (2011). Multi-dimensional optimal order detection (mood) — a very high-order finite volume scheme for conservation laws on unstructured meshes, 6th Finite Volume and Complex Application, Prague, Czech Republic, pp. 263-271. 


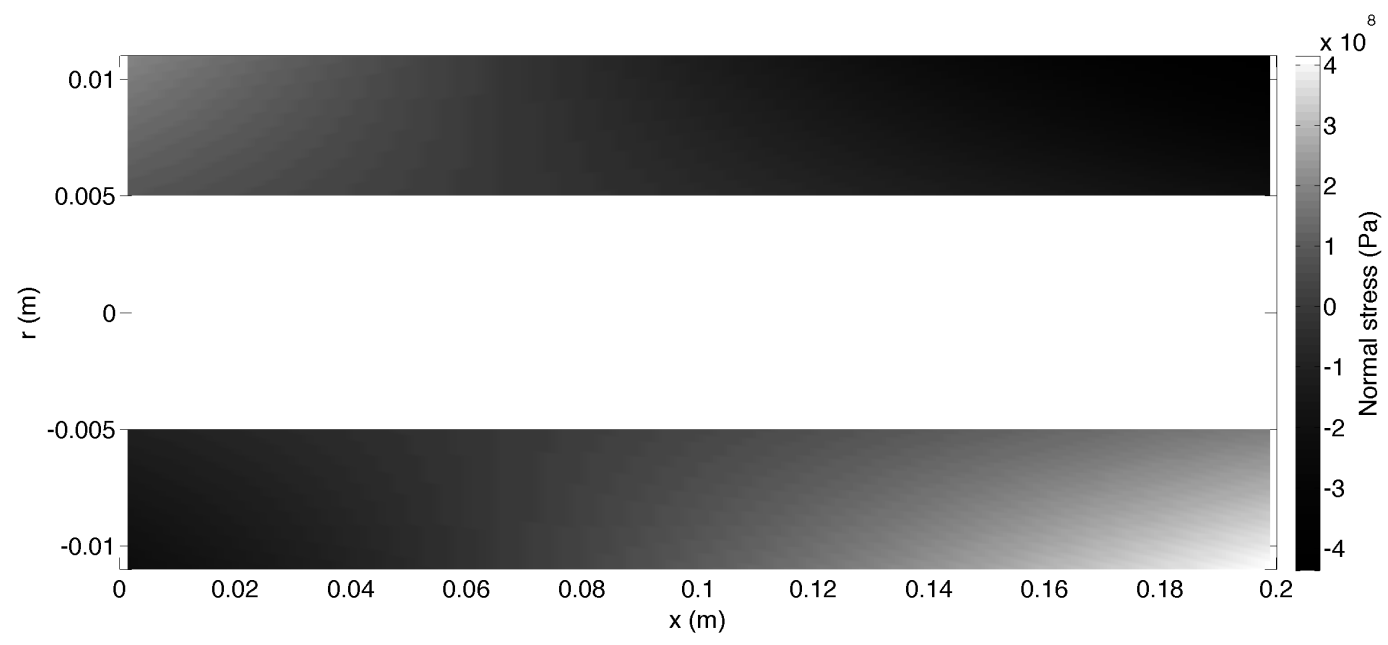

Fig. 5. Stress field of the longitudinal section of the intramedullary nail.

Dumbser, M. and Munz, C.-D. (2007). On source terms and boundary conditions using arbitrary high order discontinuous Galerkin schemes, International Journal of Applied Mathematics and Computer Science 17(3): 297-310, DOI: 10.2478/v10006-007-0024-1.

Eymard, R., Gallouët, T. and Herbin, R. (2000). The finite volume method, in P. Ciarlet and J.L. Lions (Eds.), Handbook for Numerical Analysis, North Holland, Amsterdam, pp. $715-1022$.

Hernández, J. (2002). High-order finite volume schemes for the advection-diffusion equation, International Journal for Numerical Methods in Engineering 53(5): 1211-1234.

Kroner, D. (1997). Numerical Schemes for Conservation Laws, Wiley-Teubneur Publishers, Chichester.

Leveque, R.J. (2002). Finite Volume Methods for Hyperbolic Problems, Cambridge Texts in Applied Mathematics, Cambridge University Press, Cambridge.

Ollivier-Gooch, C. and Altena, M.V. (2002). high-order-accurate unstructured mesh finite-volume scheme for the advection-diffusion equation, Journal of Computational Physics 181(2): 729-752.

Ramos, A. and Simoes, J.A. (2009). Caracterização de cavilhas de fixação intra-medular de estabilização de fracturas ósseas, Revista da Associação Portuguesa de Análise Experimental de Tensões 17: 49-55.

Toro, E. (2009). Riemann Solvers and Numerical Methods for Fluid Dynamics, Springer, Berlin/Heidelberg.

Toro, E. and Hidalgo, A. (2009). Ader finite volume schemes for nonlinear reaction-diffusion equations, Applied Numerical Mathematics 59(1): 73-100.

Trangenstein, J.A. (2009). Numerical Solution of Hyperbolic Partial Differential Equations, Cambridge University Press, Cambridge.

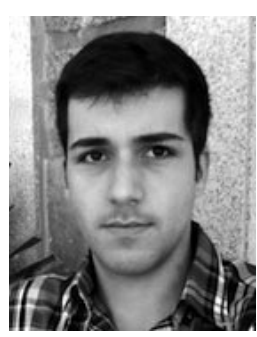

Ricardo Costa is an M.Sc. student and a researcher at the Mathematical Center of Minho University. His research interests include numerical simulation with finite volume schemes and their application to engineering problems.

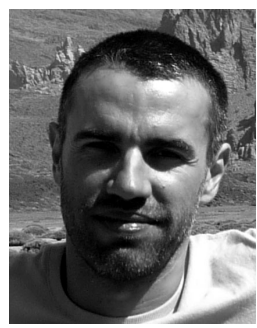

Gaspar J. Machado is an assistant professor at the Department of Mathematics and Applications of the University of Minho in Portugal. His current research interest is mainly in scientific computing, namely, the development of new finite volume schemes.

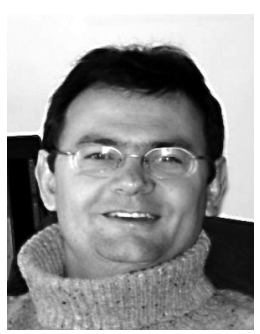

Stéphane Clain is an associate professor at the Mathematical Center of Minho University (Portugal). He works on scientific computing and numerical simulation, more specifically on the design of very high order finite volume schemes. He also develops software and a scientific library for institutional or industrial applications.

Received: 21 October 2013

Revised: 31 July 2014 